\title{
A Comparison of Alarm Treatment Results for Brazilian Children with Enuresis Plus ADHD x no ADHD
}

\author{
Carolina Ribeiro Bezerra de Sousa ${ }^{1}$, Guilherme Rodrigues Raggi Pereira ${ }^{1}$ and Edwiges Ferreira de Mattos \\ Silvares ${ }^{1,2}$ \\ 1. Behavioral Psychology Laboratory, Psychology Institute, University of São Paulo, Brazil \\ 2. Clinical Psychology Department, Psychology Institute, University São Paulo, Brazil
}

\begin{abstract}
Although the relation between enuresis (NE) and ADHD is frequently reported in the literature, the disparity among the results among several studies raises hypothesis to justify this; distinctions of types of ADHD help to understand these differences. Models for explanation of the co-occurrence, raised in a non-exhaustive literature review are displayed and analyzed in this paper as well as the report of impairments to treatment in children with nocturnal enuresis plus ADHD. The present study compares full spectrum alarm treatment plus management of other complaints besides NE (as requested by participants) of a sample of two groups of Brazilian children: with nocturnal enuresis plus ADHD (EG) versus nocturnal enuresis only (CG), searching for differences in the initial success criteria achievement time and adherence (measured directly by rate of dropouts and indirectly by lack of information from patients and by requests for management of other complaints, besides NE. With homogeneity regarding socio-demographic and clinical variables between pretreatment groups and leaving both groups free to ask for management of other complaints, no differences were observed in treatment results or time to acquire control. Comorbidity implied only in a higher proportion of lack of information from the patients. Only this indirect measure of adherence implied more failures on the treatment independently of the comorbidity.
\end{abstract}

Key words: Enuresis; ADHD; comorbidity.

\section{Introduction}

Enuresis or bedwetting is a very frequent problem among children older than five years old [1] and has social and psychological impact on those who have it [2]. This common problem can be classified as (1) primary or secondary, depending if the children have never been able to control micturition or acquired control over it for at least 6 months and then lost; (2) simple or multiple, depending if "wettings" occur only once or twice or more during the nights, and (3) non-monosymptomatic (NMNE) or monosymptomatic (MNE), depending if there are or not other problems in the lower urinary tract [3]. Houts [4] has pointed that primary, simple and MNE are more frequent than their counterparts.

Corresponding author: Edwiges Ferreira de Mattos Silvares, professor, research fields: clinical psychology. E-mail: efdmsilv@usp.br.
Epidemiological surveys undertaken in European countries suggest that the prevalence of nocturnal enuresis is $9 \%$ to $19 \%$ at 5 years, $7 \%$ to $22 \%$ at 7 years, $5 \%$ to $13 \%$ at 9 years, and $1 \%$ to $2 \%$ at 16 years [5]. A questionnaire survey of 10,960 children in the United States, however, reported somewhat higher prevalence rates of $33 \%$ at 5 years, $18 \%$ at 8 years, $7 \%$ at 11 years, and $0.7 \%$ at 17 years [6]. Yet the different criteria used in the various studies may restrict the comparability of rates of Enuresis among different countries. It is well known that this problem is up to 2 times more common in boys than it is in girls [5] what has been confirmed in one assessment study done in a Brazilian clinical context [7].

Studies on the prevalence and severity of enuresis in the Brazilian population are needed; only two studies carried out with a probabilistic sample in an urban center (in southern Brazil) were found by the present authors, in a non-exhaustive review. Study of Mota, 
Victora e Hallal [8] reported 20.1\% prevalence rate of nocturnal enuresis in boys and $15.1 \%$ in girls, aging between 3 and 9 years, and based on the criterion of one wetting episode per night. Schoen-Ferreira et al. [9] reported 11.3 prevalence rates at 6 to 10 years, $6.6 \%$ at 11 an 14 years and $1.8 \%$ at 15 and 18 years.

It should be added that no different scenery is found about treatment of this problem in Brazil, compared to many other countries. It's known that treatments for enuresis include urine alarms, decompression and tricyclic antidepressants that are used either as monotherapy or in combination, and the alarm therapy is one of the first line treatments for enuresis [10]. So far in Brazil, only Pereira, Braga \& Silvares [11] research was done on the efficacy of alarm treatment used together with weekly psychological support sessions for individual families or groups. Their sample of 84 Brazilian children and adolescents with nocturnal enuresis reached $71 \%$ of initial success (14 "dry nights") and $29 \%$ of failures (with dropouts), confirming international data, which varies between 67-80\% (e.g., Özgür et al., 2009 [1], in Turkey; Gim, Lillystone \& Caldwell, 2009 [12], in Australia, with rate of failures respectively $32 \%$ and $27 \%$ ).

After the effectiveness of treatment with urine alarm has already been demonstrated [3], research has focused on the analysis of moderating variables, which influences in the results, such as socio-demographic and clinical variables.

Our interest rests precisely in investigating the interference of comorbidity of Attention Deficit Disorder and Hyperactivity (ADHD) and Enuresis. The association between bedwetting and ADHD is frequently reported in both directions. Approximately $20-30 \%$ of children with enuresis show also ADHD [13]. The opposite, that is, presence of ADHD in a population of individuals with enuresis, has several diverse results in literature: from about $10 \%[14,15]$ to $30 \%$ [13]. Baeyens et al. [16] distinguish that prevalence in accordance with the subtypes of ADHD, finding out $15 \%$ of the combined ADHD and $22.5 \%$ of the ADHD with predominant attention-deficit, which would justify the divergence between studies.

Despite the comorbidity between enuresis and ADHD being highly commented, the causes of this association are still not well understood. In the last years there was an effort directed to reach this understanding, as it's known that the questions of causality are only speculative. Four hypotheses are plausible as causal explanations for the comorbidity between enuresis and ADHD:

(1) the relation between enuresis and ADHD may be understood by subjacent risk factors, such as gender (males), social disadvantage and maturational development delay enuresis. This one is the mostly endorsed in literature, since enuresis and ADHD show some common risk factors, such as gender (male), social disadvantage $[15,17]$ and maturational development delay [18, 19].

(2) enuresis can precede and induce ADHD: Fergusson and Horwood [20] demonstrated then that children that "wetted" their beds after 10 years of age attained slightly higher scores on rates of conduct problems and ADHD at age 13, and of anxiety and depression at age 15. Such results suggest that the non-treatment of enuresis can act as a predictor of future psychiatric pictures, including ADHD.

(3) ADHD can induce enuresis: in agreement with Baeyens et al. [21], a brain system dysfunction of children with ADHD of the inattentive type exerts a negative effect on the cerebral excitability and signalization of vesical filling, thus impairing the acquisition of sphincter control, explaining the fact that this population is more resistant to treatment.

(4) no causal relation is present, thus the relation among the two concepts is based only in casualty, reinforced by genetic studies that point the independency of the disorder transmission mode, being improbable a common genetic basis between those problems.

Although the relation between enuresis and ADHD is yet not sufficiently clear, with findings that point out 
for many possible ways, is consensus that the superposition of disorders amplifies the difficulties faced by the child, which ends displaying more behavioral problems. It is already known that individuals with enuresis display higher rates of behavioral problems when compared to controls, and lower when compared to clinical samples (e.g., Friman et al., 1998 [22]; Santos \& Silvares, 2007 [23]). Few of these studies, however, compare an "enuresis population" with a "psychiatric population". One of these few is that of Baeyens et al. [16], that revealed being the children with enuresis and ADHD the displayers of more internalizing and externalizing behavior problems than those with enuresis only. In the same direction, but facing specifically psychiatric disorders, in the study of Ghanizadeh [24] children with ADHD and enuresis were significantly more diagnosed with ODD than did controls - children with ADHD only.

Other study on this comorbidity is the Zavadenko, Kolobova and Surinova [25] that reported, in turn, that the presence of enuresis in patients (6 to 14 years) with ADHD was associated with a significant increase in the incidence of comorbid disorders, and presence of anxiety is the most common. The only parental psychiatric disorder that was related to the groups was father's major depressive disorder. This leads to conclude that enuresis and ADHD comorbidity may have a relationship with ODD and physicians who treat this kind of patients should routinely inquire about the presence of major depression in the fathers.

The implications of the presence of comorbidity in the treatment of enuresis are also an issue not sufficiently clear, because there are few investigations in the literature about it. When present, the investigation is usually about differences between pharmacological treatments, such as in Chertin et al. [26]. In this study, the authors tested the combination of DDAVP plus oxybutynin versus imipramine and observed the superiority of the first intervention to decrease the number of "wettings". The study did not compare, however, the effectiveness of the intervention in contrast to a control group. This design was made by Crimmins, Rathbun \& Husmann [27], which in convergence with the objective of the present study used the urine alarm.

Crimmins et al. [27] in a retrospective study, has reviewed protocols of treated patients with ADHD, urinary incontinence (nocturnal enuresis), and has reported a statistically significant impairment to the treatment results for children that have NE and ADHD, related to a lesser adherence to the behavioral procedures of intervention, in their own words for noncompliance. According to the authors, two factors impact the resolution of wetness in patients with ADHD - treatment noncompliance and lower IQ. Specifically their findings were: the presence of ADHD had a negative effect on reaching control, with $68 \%$ of the comorbidity patients becoming "dry" compared to $91 \%$ of controls (no ADHD) Besides that, $48 \%$ of the patients with ADHD did not adhere to the procedures in contrast to $14 \%$ of controls The comparison between long-term results of different treatments in their study is also striking since Crimmins et al. [27] data have shown that patients with ADHD and NE responded similarly to controls when using desmopressin and imipramine but were less likely to be continent after six months the initial treatment (43\% versus $69 \%, \mathrm{p}<0.01)$ and of displaying a durable response following management with an urine alarm $(19 \%$ versus $66 \%, p=0.01)$. Facing these negative findings the authors conclude that "current treatment modalities used for the child with ADHD and urinary incontinence are inadequate" and suggested that "alterations of current treatment protocols are merited, perhaps by the addition of a child psychologist to form a therapeutic team to treat the patient with ADHD and urinary incontinence".

In order to contribute to this last theme, the present study compares results of alarm treatment for Brazilian children with enuresis plus ADHD in contrast to children with enuresis without ADHD in a model of 
treatment that includes psychological sessions as support for the family. In this process it was evaluated the total time needed for the patients of both groups to achieve the initial success and differences in adherence. While drop-outs were seen as direct measures of low adherence, non-delivery of clinical records in the sessions were seen as indirect measures.

Our hypotheses were that changes on treatment protocols could promote adherence to procedures required for managing the difficulties and thus get better results. It is believed that children with comorbidity will spend more time in treatment, as they may have greater problems in following rules and displayed other problems that may also be targeted.

\section{Method}

\subsection{Participants}

The inclusion criteria used in this research were: age between 6-13 years; clinical evidence of primary nocturnal enuresis with no diurnal incontinence or encopresis; the participant should not already be in any treatment for enuresis resolution, be it psychotherapy or pharmacological intervention; the child and your caretaker should be available to assist to the biweekly therapy sessions; the Consent Term had to be signed. With these parameters, two groups were formed: the experimental group - EG (enuresis plus ADHD; $\mathrm{n}=$ 13), and the control group - CG (enuresis only; $\mathrm{n}=$ 18). Children in the EG had DSM-IV-based ADHD diagnosis, some of them were on ADHD medication (Methylphenidate) and presented another externalizing disorders in co-occurrence, such as Conduct Disorder, Opposing-Defiant Disorder and/or Bipolar Mood Disorder, but these were not the predominant disorders. Children with psychotic disorders or invasive developmental disorders were excluded. These participants were recruited either from an ADHD psychiatric treatment service, having its diagnostic made by a specialized psychiatrists, or from the enuresis treatment service.

\subsection{Materials and Equipment}

Bell-and-pad alarms lent to families by the Laboratory of Behavioral Therapy, from the Institute of Psychology of the USP (IPUSP) were used in treatment. The apparatus is composed of a $9 \mathrm{~V}$ battery-powered sound unit, tied by a wire to a large plastic sheet with aluminum stripes. The alarm device has an on-off switch that is controlled by the child and a small red led to help find the device at night. When the "pad" gets moist with urine, the "bell" emits a loud, intermittent beep, accompanied by the led flash; to stop, the child must awake and turn off the equipment.

\subsection{Evaluation Instruments}

Brazil Criterion (ABEP, 2008) [28] - To assess urban families socioeconomic status: it is based on the number of durable goods such as household items and vehicles, and other factors as the educational level of the head of the family, that are converted into points. The sum of these, which ranges from 0 to 46 , allows the classification of "economic classes", in crescent order of socioeconomic status: E (0-7), D (8-13), C2 (14-17), C1 (18-22), B2 (23-28), B1 (29-34), A2 (35-41), and A1 (42-46).

"Wettings" record: Each session was provided a new record to be completed during the fortnight and the children were asked to keep biweekly records regarding their "dry" and "wet nights" and, with parental supervision, other relevant information as the size of the wet patch in the bed, when the episode occurred during the night, if the child failed to awake to the alarm sound and if there's compliance with the proposed procedures.

Enuresis Assessment Form (Blackwell, 1989) [29]

- Semi structured clinical interview that allows one to assess the diagnostic hypothesis of enuresis on the following items: nature of the problem (primary $\mathrm{x}$ secondary), comorbidity of encopresis and other symptoms of the lower urinary tract, family history of enuresis, severity of the problem (number of wettings per week) and prevention/coping strategies from the 
parents (waking the child periodically during the night, fluid restriction, rewards or punitions after waking dry or wet); there is also a medical record, with data of any illnesses and medications taken by the child.

\subsection{Intervention Procedures}

The same treatment was offered to the families of the EG and the CG and lasted up to 32 weeks - it was stopped on the deadline or if the child had reached success criteria on an earlier period. Aiming at an outpatient model, the psychological sessions were carried out fortnightly, lasted about 30 minutes, included the child and your caretaker, and followed the model proposed by the Full Spectrum Home Training [30]. This consists of using the urine alarm associated with additional activities, such as retention control training, sphincter control training, cleanliness training, food restriction (only food with diuretic effect near bedtime, such as citric fruits juice, coffee, tea and other bladder irritants) and overlearning (relapse prevention procedure).

The fortnightly sessions of psychological care had as main objectives to monitor progress in the use of urine alarm and modifying contingencies to favor the instruction following. It also served to intervene on other childhood problems reported by parents.

Classification Criteria for the treatment results (based on Butler, 1991 [31]): Initial Success: obtainment of 14 consecutive "dry nights", but failing the overlearning procedure. Discharge: reaching the initial success criteria and completing overlearning. Lack of sucess: failure to achieve the initial success criteria in 32 weeks. Drop-Out: if the family missed two consecutive meetings or declared abandonment of treatment.

\subsection{Statistical Analyses}

For statistical comparison a control population referred for NE during the same time interval was treated. The only criteria to be in the control group was to have NE but medical diagnosis of ADHD. It was verified, later on, that both groups matched for, gender, clinical variables, enuresis types and SES. Statistical evaluations used Exact Fisher test with $\mathrm{p}<0.05$ were considered significant.

\section{Results}

Table 1 shows the percentual distribution of the participant's sociodemographic and clinical variables for both experimental and control groups.

The pre-treatment homogeneity between the groups has been verified in relation to the socio-economic and clinical variables in Table 1. No pretreatment differences were found between groups in gender, socioeconomic status, type of enuresis (MNE x NMNE and simple $\mathrm{x}$ multiple) and number of "wettings" per week, which has not been mentioned in the literature.

Shown on Fig. 1 are the results related to acquisition of vesical control (initial success and discharge), non-acquisition (lack of sucess) and drop-outs for both groups. An independence test showed no difference between groups (GE x GC) on these treatment results $(p=0.645)$. As can be seen in Fig. 1 both groups reached a high proportion of "discharges", which is a good prognostic in regard to relapse.

The Fig. 2 shows the "Initial Success" risk curve for both groups along 32 weeks of treatment. Inspection of the Fig. 2 make evident the equality in response acquisition time in both groups $(p=0.964)$ in a log rank analysis, what seems to contradict one of our hypothesis.

Table 2 shows the comparison between groups concerning the number and the percentage of clients that fail to provide information for the therapist (not delivering the records) — indirect measures of adherence - and request handling for other concerns. As it can be seen in Table 2, only one of these measures (not providing information) could distinguish the groups. Comorbidity implied a higher proportion of lack of information, but not higher request for management of other complaints. 
Table 1 Socio-demographic and clinical variables: descriptive statistics data.

\begin{tabular}{|c|c|c|c|}
\hline & EG & CG & p value \\
\hline \multicolumn{4}{|c|}{ Socio-Demographic Variables } \\
\hline Gender & Percentage (n) & & \multirow{4}{*}{0.095} \\
\hline Male & $92.3 \%(12)$ & $61.1 \%(11)$ & \\
\hline Female & $7.7 \%(1)$ & $38.9 \%(7)$ & \\
\hline Total & $100 \%(13)$ & $100 \%$ & \\
\hline \multicolumn{4}{|c|}{ Socio-Economic Status } \\
\hline Mean (SD) & $30.2(6.18)$ & $28.8(8.09)$ & \multirow{2}{*}{0.567} \\
\hline Min-max & $20.0: 41.0$ & $13.0: 41.0$ & \\
\hline \multicolumn{4}{|c|}{ Clinical Variables - ENURESIS } \\
\hline Simple & $69.2 \%(9)$ & $72.2 \%(13)$ & \multirow{3}{*}{1.000} \\
\hline Multiple & $30.8 \%(4)$ & $27.8 \%(5)$ & \\
\hline Total & $100 \%(13)$ & $100 \%(18)$ & \\
\hline \multicolumn{4}{|c|}{ Enuresis: Monosymptomatic X Non-Monosymptomatic } \\
\hline Monosymptomatic & $61.5 \%(8)$ & $88.9 \%(16)$ & \multirow{3}{*}{0.099} \\
\hline Non-Monosymptomatic & $38.5 \%(5)$ & $11.1 \%(2)$ & \\
\hline Total & $100 \%(13)$ & $100 \%(18)$ & \\
\hline \multicolumn{4}{|c|}{ Intial Frequence - “Wettings per Week" } \\
\hline Mean (SD) & $5.35(1.709)$ & $5.99(1.472)$ & \multirow{2}{*}{0.211} \\
\hline Min-max & $2.00: 7.00$ & $2.00: 7.00$ & \\
\hline
\end{tabular}

Table 2 Flow of the psychological attendance - variable analysis.

\begin{tabular}{|c|c|c|c|}
\hline & EG & CG & p value \\
\hline \multicolumn{4}{|c|}{ Lack of Information (non-delivery of the records at the session) } \\
\hline Yes & $53.8 \%(7)$ & $16.7 \%(3)$ & \\
\hline No & $46.2 \%(6)$ & $83.3 \%(15)$ & 0.050 \\
\hline Total & $100 \%(13)$ & $100 \%(18)$ & \\
\hline \multicolumn{4}{|c|}{ Requests for Management of Other Complaints } \\
\hline Yes & $46.2 \%(6)$ & $16.7 \%(3)$ & \\
\hline No & $53.8 \%(7)$ & $83.3 \%(15)$ & 0.114 \\
\hline Total & $100 \%(13)$ & $100 \%(18)$ & \\
\hline
\end{tabular}

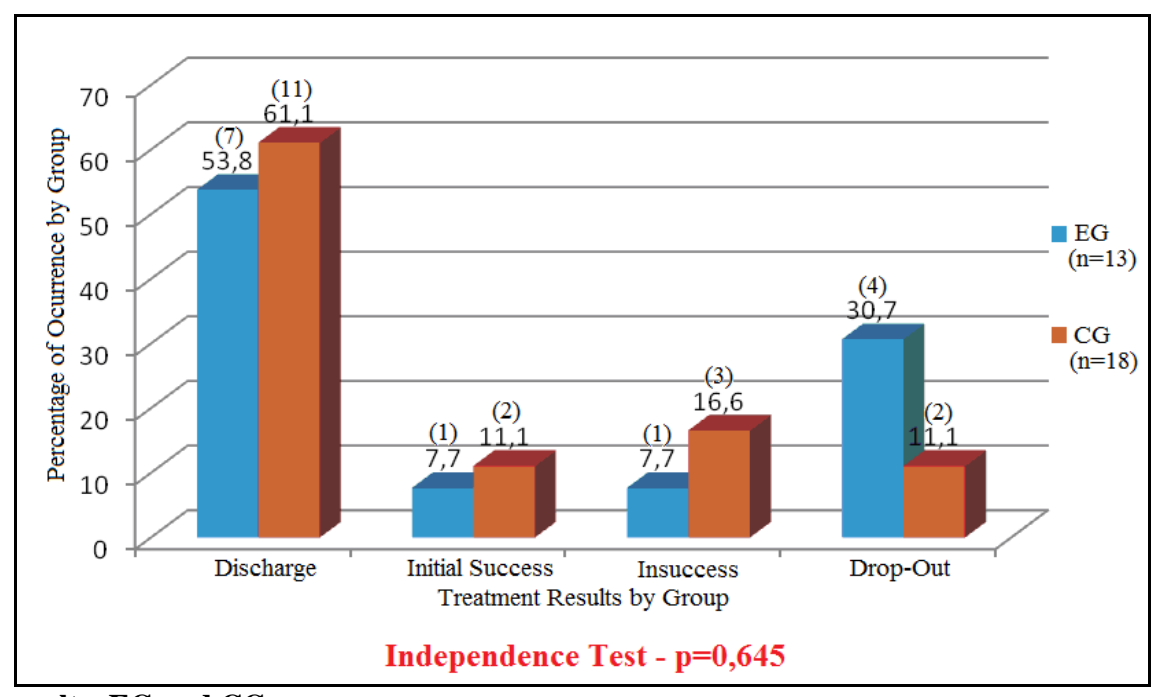

Fig. 1 Treatment results: EG and CG. 


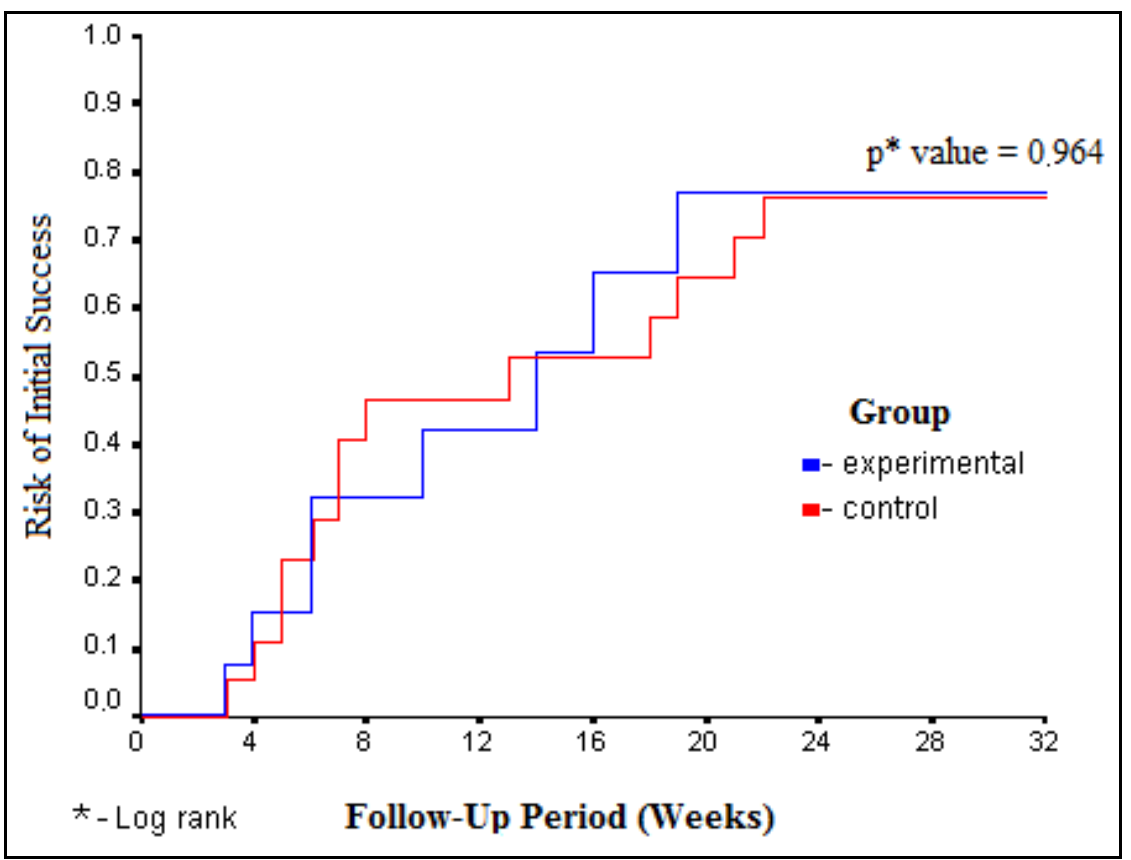

Fig. 2 Risk curve: initial success.

Although the two groups were similar in regard to treatment outcomes separately, when considering the whole group of participants $(\mathrm{n}=31)$, an association between failing to provide information and failure results (lack of sucess + drop-outs) was found. In other words, low adherence of the client was associated with more negative results in treatment, independently to what group this lack of adherence is referred to $(\mathrm{p}=$ 0.004 in the Independence Test).

\section{Discussion}

Mentioning number of dropouts as separated from number of lack of success outcomes in the treatment as we do in this study is not very common in the enuresis literature; most of studies mention only initial success and some give also failures rates. This fact makes difficult the comparison between the present study results in terms of dropouts with the ones from other studies on treatment of enuresis, with urine alarm international studies as Özgür et al. [1] and Gim et al. [12], for example. Even in the only Brazilian study cited before this rate of dropouts were not mentioned. We suppose in all studies of enuresis treatment, dropouts plus lack of sucess rates were presented together. So extracting from their initial success rate their rate of failures we can say that our rate of success or failures fall in the same range.

As expected, our $72.2 \%$ of success for $\mathrm{CG}$ (discharge plus initial success) is similar to international rates for treatment of enuresis and $61.5 \%$ for GE is what was obtained by Crimmins et al. [27] in treatment of enuresis with ADHD. In other words, this data confirms the mean index of efficacy of alarm intervention in international studies as well as in the only Brazilian study with alarm found [11].

The possibility to manage other complaints than NE in this study also made decrease drop-out rates in the EG and brought no statistic significance in the independence test $(p=0.645)$ as found by Crimmins et al. [27]. So, changes in treatment protocol were not associated to lower rates of dropouts for $\mathrm{GE}$ in comparison to GC. The lack of a discriminative effect of ADHD was probably determined by the management of other complaints by the clinical psychologist. However as GE and GC were able to do this management it is not possible to attribute with confidence any differences between groups to this variable. 
Yet, as somehow expected by our hypothesis, comorbidity implied a higher proportion of lack of information, but not higher request for management of other complaints, what is unexpected. The fact that the indirect index of non-adherence to treatment is associated with bad results independent of presence of ADHD might be what really should require attention from researchers in the future.

We can speculate that what makes the present study closer to the one of Crimmins et al. [27] is the fact that these authors advice changes in the treatment protocol with the help of a clinical psychologist as necessary to help children with NE plus ADHD, what has been done in the present study. Considering our results we advice for enuresis treatment careful attention to other complaints besides NE, independent of comorbidity with ADHD, especially in function of the homogeneity between groups concerning the management of other problems. We hypothesize that was the lack of adherence (directly and indirectly measured here) only directly measured by Crimmins et al. [27] study is the most important point researchers have to focus.

Since Crimmins et al. [27] research did not analyze indirect measures of adherence, this last assertion has to be taken cautiously. However, it is important recalling that psychological attendance was given in the sessions to manage eventual contingencies individually, in order to increase the adherence for both groups. This can be thought as a way for minimizing differences between groups, dealing with possible variables that could lead to a result less favorable for the EG what again might turn impossible the comparison between this and the Crimmins et al. [27] study. Yet, as the therapist and the researcher were the same person, that could be biased on the obtained distinction between groups; in other words, this could be a confounding variable.

Another important point to mention that brings low confidence on this contradiction is the small size of both groups. For practical considerations (small sample of NE with comorbidity) we could not from the start of the study to match the two groups in relation to socio-demographic and clinical variables. It was providential and striking the statistical homogeneity between GE e GC in gender, socio-economic disadvantage and clinical variables; none study so far has mentioned this fact.

It is difficult to assure reliably any relation when the sample is not large enough as is the case here. This size however was reached not so easily, what brings some doubts to the authors about the rates of comorbidity among the two disorders found in literature. Considering that all profiles of all inpatients, enrolled from 2008-2009 in one Brazilian ADHD center and in one Brazilian Enuresis Center were examined to find comorbidity cases, this founding number of participants for the present study is way below the expected. Other studies on the consequences for treatment of Enuresis with ADHD association as well as epidemiological ones about this comorbidity should be done to bring more confidence to our present results and interpretations.

With the same aim, it would be relevant in the future study, to include control about other variables, such as measures of IQ, present in study of Crimmins et al. [27]. Considering the study of Zavadenko et al. [25], it would be also relevant to include the relationship of ODD and parents with depression, points still to be open to investigation.

\section{References}

[1] B. C. Özgür, S. Özgür, V. Doğan and U. A. Örün, The efficacy of an enuresis alarm in monosymptomatic nocturnal enuresis, Singapore Med J. 50 (9) (2009) 880.

[2] G. C. Coppola, M. Gaita and D. Saraulli, Psychological correlates of enuresis: A case-control study on an Italian sample, access in June 1st, 2011, available online at: http://www.springerlink.com/content/hn1q65k221971jr1/.

[3] T. Nevéus, A. von Gontard, P. Hoebeke, S. Bauer, W. Bower, J. V. Walle, C. K. Yeung, J. C. Djurhuus, The standardization of terminology of lower urinary tract function in children and adolescents: report from the Standardisation Committee of the International Children's Continence Society, The Journal of Urology 176 (1) (2006) 314-324. 
[4] A. C. Houts, Behavioral treatment for enuresis, in: A. E. Kazdin and J. R. Weisz, Evidence-based Psychotherapies for Children and Adolescents, New York: The Guilford Press, 2010, pp. 359-374.

[5] R. J. Butler, Annotation: Night wetting in children: psychological aspects, Journal of Child Psychology and Psychiatry 39 (4) (1998) 453-463.

[6] R. S. Byrd, M. Weitzman, N. E. Lanphear and P. Auinger, Bed-wetting in US children: Epidemiology and related behavior problems, Pediatrics 98 (3) (1996) 414.

[7] D. R. Emerich, C. R. Sousa and E. F. Silvares, Estratégias de enfrentamento parental e perfil clínico e sociodemográfico de crianças e adolescentes com enurese, Revista Brasileira de Crescimento e Desenvolvimento Humano 21 (2011) 240-250.

[8] D. M. Mota, C. G. Victora and P. C. Hallal, Investigation of voiding dysfunction in a population-based sample of children aged 3 to 9 years, Jornal de Pediatria 81 (3) (2005) 225-232.

[9] T. Schoen-Ferreira, M. Marteleto, E. Medeiros, M. Fisberg and M. Aznar-Farias, Levantamento de enurese no município de São Paulo, Revista Brasileira de Crescimento e Desenvolvimento Humano 17 (2) (2007) 31-36.

[10] T. Nevéus, P. Eggert, J. Evans, A. Macedo, S. Rittig, S. Tekgül, J. Vande Walle, C. K. Yeung and L. Robson, Evaluation and treatment of monosymptomatic enuresis - A standardisation document from the International Children's Continence Society (ICCS), 2010.

[11] R. F. Pereira, P. Braga and E. F. M. Silvares, Behavioral alarm treatment for nocturnal enuresis in a Brazilian sample. International Braz J Urol (Printed) 36 (2010) 332-338.

[12] C. S. Y. Gim, D. Lillystone and P. H. Y. Caldwell, Efficacy of the bell and pad alarm therapy for nocturnal enuresis, Journal of Paediatrics and Child Health 45 (7-8) (2009) 405-408.

[13] J. Biederman, S. L. Santangelo, S. V. Faraone, K. Kiley, J. Guite, E. Mick, E. D. Reed, I. Kraus, M. Jellenek and J. Perrin, Clinical correlates of enuresis in ADHD and non ADHD children, Journal of Child Psychology and Psychiatry 36 (5) (1995) 865-877.

[14] A. Ghanizadeh, M. R. Mohammadi and R. Moini, Co-morbidity of psychiatric disorders and parental psychiatric disorder of attention deficit hyperactivity disorder children, Journal of Attention Disorders 12 (2008) 149-155.

[15] S. Shreeram, J. P. He, A. Kalaydjian, S. Brothers and Merikangas, Prevalence of enuresis and it is association with attention-deficit/hyperactivity disorder among US children: results from a nationally representative study,
Journal of American Academy of Child and Adolescent Psychiatric 48 (2009) 35-41.

[16] D. Baeyens, H. Roeyers, P. Hoebke, S. Verté, E. Van Hoecke and J. V. Walle, Attention deficit/ hyperactivity disorder in children with nocturnal enuresis, Journal of Urology 171 (2004) 2576-2579.

[17] E. Van Hoecke, D. Baeyens, J. Vande Walle, P. Hoebeke and $\mathrm{H}$. Roeyers, Socioeconomic status as a common factor underlying enuresis and psychopathology, Journal of Developmental and Behavioral Pediatrics 24 (2003) 109-114.

[18] R. J. Butler, Childhood nocturnal enuresis: Developing a conceptual framework, Clinical Psychology Review 24 (2004) 909-931.

[19] P. Sureshkumar, M. Jones, P. H. Y. Caldwell and J. C. Craig, Risk factors for nocturnal enuresis in school-age children, The Journal of Urology 182 (6) (2009) 2893-2899.

[20] D. M. Fergusson and L. J. Horwood, Nocturnal enuresis and behavioral problems in adolescence: A 15 years longitudinal study, Paediatrics 94 (1994) 662-667.

[21] D. Baeyens, H. Roeyers, S. Naert, P. Hoebke and J. V. Walle, The impact of maturation of brainstem inhibition on enuresis: A startle eye blink modification study with 2-yearfollowup, The Journal of Urology 178 (2007) 2621-2625.

[22] P. C. Friman, M. L. Handewerk, S. M. Swearer, J. C. McGinnis and W. J. Warzak, Do children with primary nocturnal enuresis have clinically significant behavior problems? Acta Pediatric Adolescence Med. 152 (1998) 537-539.

[23] E. O. L. Santos and E. F. M. Silvares, Crianças enuréticas e crianças encaminhadas para clínicas: um estudo comparativo da percepção de seus pais Psicologia: Reflexão e Crítica, Porto Alegre 19 (2007) 277-282.

[24] A. Ghanizadeh, Comorbidity of enuresis in children with Attention-Deficit/Hyperactivity Disorder, Journal of Attention Disorders 13 (5) (2010) 464-467.

[25] N. Zavadenko, N. Kolobova and N. Y. Suvorinova, Attention deficit hyperactivity disorder and enuresis in children and adolescents, Neuroscience and Behavioral Physiology (2011) 1-7.

[26] B. Chertin, D. Koulikov, W. Abu-Arafeh, Y. Mor, Z. O. Shenfeld and A. Farkas, Treatment of nocturnal enuresis in children with attention déficit hyperactivity disorder, Journal of Urology 178 (4) (2007) 1744-1747.

[27] C. R. Crimmins, S. R. Rathbun and D. A. Husmann, Management of urinary incontinence and nocturnal enuresis in attention-deficit hyperactivity disorder, The Journal of Urology 170 (2003) 1347. 
[28] Associação Brasileira de Empresas de pesquisa. Critério de Classificação Econômica Brasil, Access in Feb. 8th, 2007, available online at: http://www.abep.or/codigosguias/ABEP_CCEB.pdf.

[29] C. Blackwell, A Guide To Enuresis: A Guide to A Treatment of Enuresis For Professionals, Bristol: Enuresis Resource and Information Center, 1989.
[30] A. C. Houts, Behavioral treatment for enuresis, in: A. E. Kazdin and J. R. Weisz (Eds.), Evidence-Based Psychotherapies for Children and Adolescents, New York: Guilford Press, 2003, pp. 389-406.

[31] R. J. Butler, Establishment of working definitions in nocturnal enuresis, Archives of Disease in Childhood 66 (1991) 261-271. 